\title{
BRYOFLORA OF THE "POHULYANKA" FOREST PARK (LVIV CITY). I. CHANGES IN TAXONOMIC COMPOSITION UNDER ANTROPOGENIC TRANSFORMATION
}

\author{
Z. Mamchur, Yu. Drach, I. Danylkiv
}

Ivan Franko National University of Lviv, 4, Hrushevskyi St, Lviv 79005, Ukraine e-mail: dzvinkamamchur@gmail.com

The article presents a list of bryophytes on the territory of the forest park "Pohulyanka" in Lviv city which includes 143 species of mosses which belong to 79 genera, 36 families of two divisions: Marchantiophyta and Bryophyta. Changes in the species composition for the last 50-100 years were analyzed: 34 species were not detected, the reduction of the species diversity of the liverworts and decreasing of the proportion of moss families: Polytrichaceae Schwägr., Thuidiaceae Schimp., Hylocomiaceae M. Fleisch. are noted. These families are changed the least: Brachytheciaceae Schimp., Hypnaceae Schimp., Mniaceae Schwägr. 72 species of bryophytes are indicated for the studied area for the first time, and Eucladium verticillatum (With.) Bruch et Schimp. is indicated for the first time for the territory of Lviv region. 25 species are rare for the nemoral and foreststeppe zones. The substrate affiliation of the bryophytes was presented. It was shown that the spectrum of substrates for the epiphytes has expanded as a result of the anthropogenic activity.

Keywords: bryophytes, anthropogenic transformation, rare bryophytes, forest park "Pohulyanka", Lviv city

\section{ABSTRACT}

The researches of the bryophlora of Lviv and its surroundings are known since the middle of the $19^{\text {th }}$ century (the oldest dates back to the publications of G. Lobazhevsky (Jobarzewski JH, 1947, 1949), I. Cherkavsky (Czerkawski J., 1868), I. Krupa (Krupa I., $1885)$, in the first half of the $20^{\text {th }}$ century, the research was continued by F. Lilienfeld (Lilienfeldówna F., 1910, 1911, 1914), A. Zmuda (Żmuda AJ, 1911, 1912) and T. Visniewski (Wiśniewski T., 1923) [16, 30]. However, there is limited data for the territories of the forest park "Pohulyanka", the majority of information was obtained from the herbarium material. In the Herbarium of Ivan Franko National University of Lviv (LW) the bryological collections of $\mathrm{H}$. Drozdowska $(\mathrm{H}$. Drozdowska, 1939) remain unpublished. The Herbarium National Museum of Natural History of the National Academy of Sciences of Ukraine (LWS) and the Institute of Ecology of the Carpathians of the National Academy of Sciences of Ukraine (LWKS) have material from the second half of the $20^{\text {th }}$

() 2018 Z. Mamchur et al.; Published by the Ivan Franko National University of Lviv on behalf of Біологічні Студії / Studia Biologica. This is an Open Access article distributed under the terms of the Creative Commons Attribution License (http://www.budapestopenaccessinitiative.org/ and Creative Commons Attribution 4.0 License), which permits unrestricted reuse, distribution, and reproduction in any medium, provided the original work is properly cited.

ISSN 1996-4536 (print) • ISSN 2311-0783 (on-line) • Біологічні Студії / Studia Biologica • 2018 • Том 12/№1 • C. 99-112 
century (A. Lazarenko, K. Ulychna, M. Slobodian, O. Vysotska, E. Lesniak) and modern (I. Danylkiv, Z. Mamchur, M. Rahulina).

The aim of the study was to summarize the author's, herbarium and literary information about the diversity of bryophytes of the forest park "Pohulyanka" and the Botanical Garden of Ivan Franko National University of Lviv (Cheremshyna St.) was to study species composition of the bryophytes, the nature of the species substrate distribution, as well as to find out the pecularities of bryoflora anthropogenic transformation over the last $50-100$ years.

\section{MATERIALS AND METHODS}

Field researches were carried out by traditional methods during the vegetation period between 2014-2017 during different seasons. All substrates on the territory of the forest park "Pohulyanka" and Botanical Garden of Ivan Franko National University of Lviv on Cheremshyna St. were examined (Fig. 1).

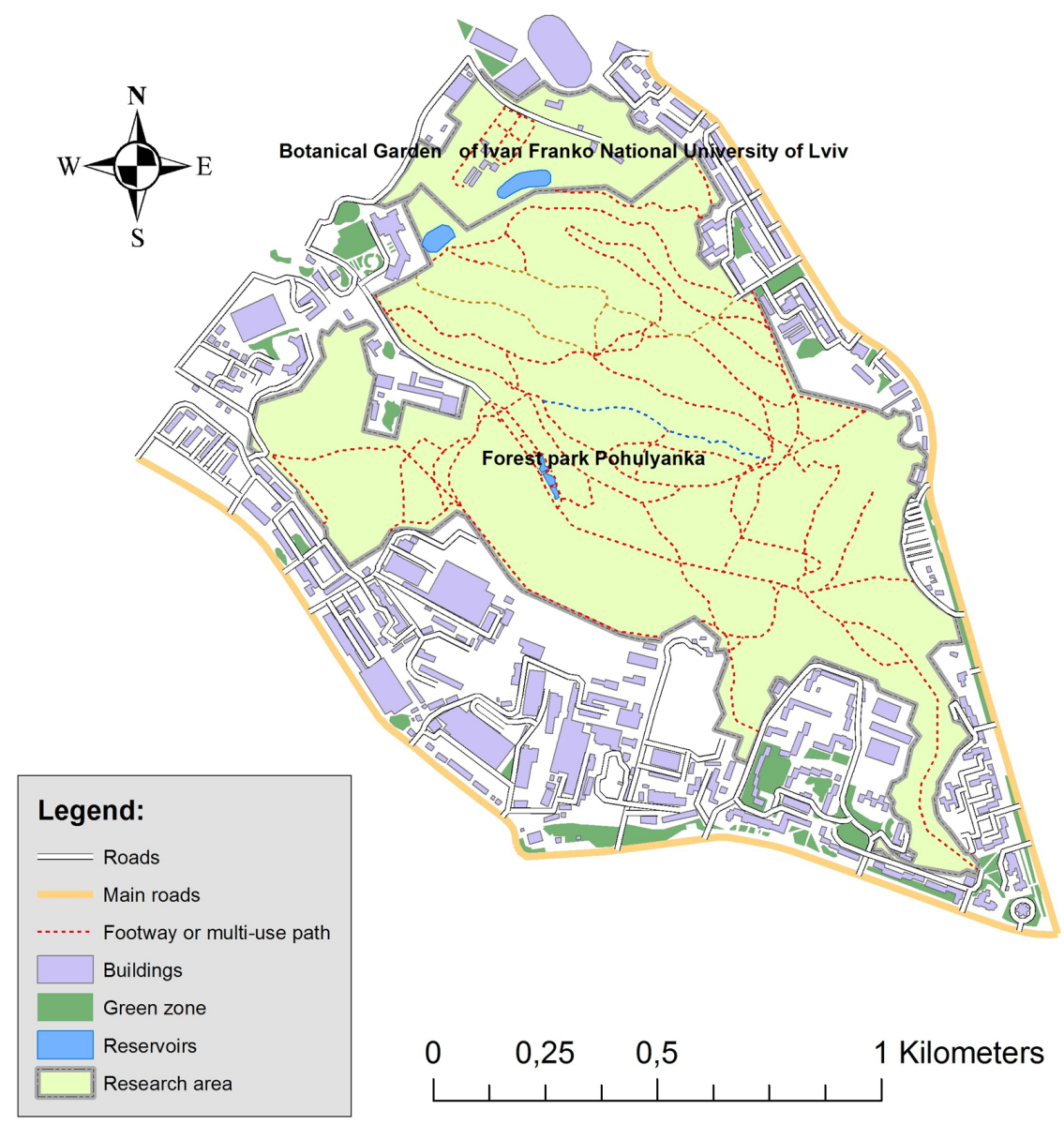

Fig. 1. The map scheme of the forest park "Pohulyanka" (Lviv city, $49^{\circ} 49^{\prime} 24^{\prime \prime} \mathrm{N} 24^{\circ} 3^{\prime} 54^{\prime \prime} \mathrm{E}$ )

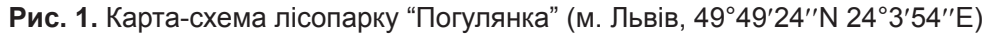

ISSN 1996-4536 (print) • ISSN 2311-0783 (on-line) • Біологічні Студії / Studia Biologica • 2018 • Том 12/№1 • C. 99-112 
The work contains the material from our own field researches, herbarium samples of the forest park "Pohulyanka" bryophytes from the H. Drozdowska (LW) and other samples which we critically worked and other samples which we critically worked through, the materials of Herbarium (LWS, LWKS), as well as literary data $[1 ; 2 ; 25 ; 31 ; 32]$.

Sampling and analysis of the material was carried out according to generally accepted methods. Latin names of taxa were given by M. Boiko (2014) [5].

The territory that we research was "Pohulyanka", located in Lychakivsky district of Lviv, as well as the adjoining area of the Botanical Gardens of Ivan Franko Lviv National University on Cheremshyna Street.

The forest park "Pohulyanka" is located in the south-eastern part of the city, on the rising of the Davydiv Ridge. The territory of this forest park has undergone significant changes over the centuries. It has been known from the seventeenth century that this area was an apiary for Lviv burgomaster Jan Atalmayer. The following century, a lawyer Frantsishek Venglinsky bought an estate in the park, besides constructing his Villa "Pohulyanka", he started to plant and grow a beech tree forest on the neighbouring hilly areas which he named Venglinsky forest till the $X X^{\text {th }}$ century. Later on, the restaurant, brickyard and wine factory were built. Since the $\mathrm{XV}^{\text {th }}$ century a large number of water sources made Pohulyanka the main supply of water to the city. In the 1930s, of the forest park "Pohulyanka" looked like an overgrown forest, due to the fact that large areas of land belonged to private owners where access was prohibited. The city park plans and layout began to be arranged in 1940 - part of the forest was uprooted and lawns were formed. In the valley below three small reservoirs were made, this became a central axis of the park [15]. In one of these small reservoirs Poltva (Pasica) begins, it had particularly clean water and was named the Silver Flow due to this. In 1940s from the main river three ponds were created [15]. In the 60s-70s, the eastern outskirts of the forest park were built up with high residential buildings, and in the 70s Pohulyanka was reconstructed. In particular, a network of paths was created for the discerning walker and areas for families to enjoy picnics. It was declared a public park in the nineteenth century and since 1972 has the status of a local garden park heritage, which occupies 129 hectares now. The main plantings in the park are beech and hornbeam, some types of birch and maple trees also occur here [15].

The Botanical Garden of Ivan Franko National University of Lviv is adjacent to the forest park "Pohulyanka", It was created in 1911 on the place of the former Cetnerivka ornamental garden on a complex relief. The Botanical garden contained slopes, raised plateau and a swampy valley with a pond. Subsequently, in the postwar period, the Botanical garden was increased in size, sandy slopes where introduced which were covered with herbs and shrubs, beech, hornbeam and oak trees were introduced in greater quantities.

\section{RESULTS AND DISCUSSION}

In general, the list of the area "Pohulyanka" bryophytes based on our research, on the materials of the Herbarium (LW, LWS, LWKS) and literary sources $[1 ; 2 ; 25 ; 32 ; 33]$ consists of 143 species of two divisions (Marchantiophyta, Bryophyta), 36 families and 79 genera (Table 1).

The best represented families (Brachytheciaceae Schimp. - 17 species, Pottiaceae Schimp. - 16, Mniaceae Schwägr. - 12, Orthotrichaceae Arn. - 10, Bryaceae Schwägr. - 9, Hypnaceae Schimp. - 9, Amblystegiaceae Kindb. - 8, Polytrichaceae Schwägr. - 7, Plagiotheciaceae (Broth.) Fleisch. - 7, Dicranaceae Schimp. - 4, Funariaceae Schwägr. - 4, 
Hylocomiaceae M. Fleisch. - 4, Thuidiaceae Schimp. - 4 species) comprise 111 species $(77.6 \%)$.

In genera spectrum of bryophytes, the number one species belong to genus Orthotrichum Hedw. - 9, Plagiothecium Schimp. - 7, Brachythecium Schimp. - 5, Mnium Hedw. - 5, Plagiomnium T. Kop. - 5, Ptychostomum Hornsch. -5 , Tortula Hedw. -5 , Bryum Hedw. - 4, Hypnum Hedw. - 4, Polytrichum Hedw. - 4) - 53 species (37 \%).

Table 1. The taxonomic composition of bryoflora of the forest park "Pohulyanka" (Lviv city) Таблиця 1.Таксономічний склад бріофлори лісопарку “Погулянка” (м. Львів)

\begin{tabular}{lccc}
\hline Taxon & Family & Genus & Species \\
MARCHANTIOPHYTA & $\mathbf{8}$ & $\mathbf{8}$ & $\mathbf{8}$ \\
MARCHANTIOPSIDA & 2 & 2 & 2 \\
Marchantiales & 2 & 2 & 2 \\
JUNGERMANNIOPSIDA & 6 & 6 & 6 \\
Pelliales & 1 & 1 & 1 \\
Metzgeriales & 1 & 1 & 1 \\
Jungermanniales & 4 & 4 & 4 \\
BRYOPHYTA & $\mathbf{2 8}$ & $\mathbf{7 1}$ & $\mathbf{1 3 5}$ \\
POLYTRICHOPSIDA & 1 & 3 & 7 \\
Polytrichales & 1 & 3 & 7 \\
TETRAPHIDOPSIDA & 1 & 1 & 1 \\
Tetraphidales & 1 & 1 & 1 \\
BRYOPSIDA & 26 & 67 & 127 \\
Diphysciales & 1 & 1 & 1 \\
Encalyptales & 1 & 1 & 1 \\
Funariales & 1 & 3 & 4 \\
Grimmiales & 1 & 2 & 3 \\
Dicranales & 4 & 8 & 12 \\
Bryales & 2 & 6 & 21 \\
Orthotrichales & 1 & 2 & 10 \\
Pottiales & 1 & 8 & 16 \\
Hypnales & 13 & 35 & 58 \\
Rhizogoniales & 1 & 1 & 1 \\
\hline
\end{tabular}

As previously mentioned, the area of "Pohulyanka" has often undergone significant changes during the last century: 1) until 1940; 2) war and post-war period and the years of fundamental reconstruction (1941-1990); 3) modern period (1991-2017). Analyzing the changes in the species composition, we observe first of all the disappearance of liverworts from the families Cephaloziaceae Mig., Lepidoziaceae R. M. Schust., Pelliaceae Klinggr. (Table 2). The decrease in the proportion of mosses families: Polytrichaceae Schwägr., Thuidiaceae Schimp., Hylocomiaceae M. Fleisch. (Fig. 2) is observed.

The least changes occurred in the families Brachytheciaceae Schimp., Pottiaceae Schimp., Hypnaceae Schimp., Funariaceae Schwägr., we observed an increasing of species number in some families: Amblystegiaceae Kindb., Bryaceae Schwägr., Orthotrichaceae Arn., Mniaceae Schwägr. 
Fig. 2. The changes in the spectrum of leading families of bryophytes during the 19002017 on the territory of "Pohulyanka"

Рис. 2. Зміни у спектрі провідних родин бріофрітів упродовж 1900-2017 рр. на території "Погулянки"

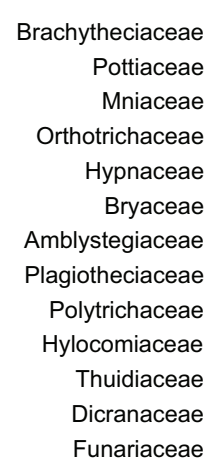

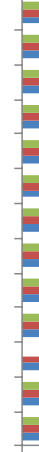

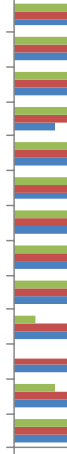

0
- 1991-2017 pp.

- 1900-2017 pp.

- 1900-1990 pp.

72 species $(50.3 \%)$ were for the first time indicated and for the researched territory. Eucladium verticillatum ${ }^{*}$ was indicated for the territory of Lviv region.

Among those bryophytes found during the century on the territory of the „Pohulyanka" forest park, no species were indicated that are included into the Red Data Book of Ukraine (2009). Physcomitrium eurystomum (Lindb. Et Arn.) Sendtn. (Funariaceae) is a species on the southern border of the European part of the disjunctive area. It is included to the Red Data Book of European Bryophytes Red ..., 1995) [22] under the category RT (Threatened Species of the Region), in the Red List of Bryophytes for Ukraine, 2010: $3^{\text {rd }}$ category (Boyko, 2010) [4], to the Checklist and country status of European bryophytes - towards a new Red List for Europe (RLfE, 2014) LC - Least Concern (there is a small threat, but the taxon can't be included in the previous categories), [14] for M. Boyko, 2015 [6]. The species was found on the territory of the Botanical Gardens of Ivan Franko National University of Lviv (Ulychna, 1964).

Among the "regionally rare" according to M. Boyko [4] 24 species are known: 11 for the Nemoral zone, 13 - Forest-Steppe. Rare for the Nemoral and Forest-Steppe zones are Acaulon muticum, Eurhynchium angustirete, Pogonatum urnigerum, Rhytidiadelphus triquetrus, which were found only in the 50s and 60s. Leucobryum glaucum, which we did not find during the last period (Table 2), occurs very often in the collection of H. Drozdovska (Drozdowska, 1939). K. Ulychna mentioned it in 1977.

The following regionally rare species have been identified for the first time in the past decades on the territory of Pohulyanka: Bryum subapiculatum, Diphyscium foliosum, Eucladium verticillatum, Fissidens exilis, Orthotrichum lyelli, Rhynchostegium confertum, Sciuro-hypnum starkei, Trichodon cylindricus.

Three zonal geographical elements (boreal, nemoral and arid) and also a cosmopolitan one in "Pohulyanka" bryoflora can be identified. Bryophyte species, belonging to the nemoral and boreal elements, have suffered from the biggest losses in recent decades, but the proportion of cosmopolites increased.

Although forest species dominate among the ecological-cenotic groups, a number of exactly forest species has disappeared: Herzogiella seligeri, Homalia trichomanoides, Hylocomium splendens, Mnium marginatum, Plagiothecium curvifolium, P. denticulatum, Pleurozium schreberi, Polytrichum piliferum, P. juniperinum, P. Iongisetum, Thuidium assimile, Th. delicatulum, Th. recognitum, including regionally rare Pogonatum urnigerum, Leucobryum glaucum, Pseudoscleropodium purum, Rhytidiadelphus triquetrus.

\footnotetext{
* Authors species are presented in Table 2
}

ISSN 1996-4536 (print) • ISSN 2311-0783 (on-line) • Біологічні Студії / Studia Biologica • 2018 • Том 12/№1 • С. 99-112 
Table 2. Species variety of bryophytes on various substrates on the territory of the forest park "Pohulyanka" (Lviv city) in different periods during 1900-2017

Таблиця 2. Видове різноманіття бріофітів на різноманітних субстратах на території лісопарку “Погулянка” (м. Львів) у різні періоди упродовж 1900-2017 рр.

\begin{tabular}{|c|c|c|c|c|}
\hline \multirow[b]{2}{*}{ Species } & \multicolumn{3}{|c|}{ Periods } & \multirow[b]{2}{*}{ Substrate } \\
\hline & $\begin{array}{c}1900- \\
1940\end{array}$ & $\begin{array}{c}1941- \\
1990\end{array}$ & $\begin{array}{c}1991- \\
2017\end{array}$ & \\
\hline 1 & 2 & 3 & 4 & 5 \\
\hline \multicolumn{5}{|c|}{ Marchantiophyta } \\
\hline Aneura pinquis (L.) Dumort. & - & - & + & SV \\
\hline Cephalozia bicuspidata (L.) Dumort. & + & - & - & so \\
\hline Conocephalum conicum (L.) Underw. & - & + & + & St, SO \\
\hline Lepidozia reptans (L.) Dumort. & + & - & - & So \\
\hline Lophocolea heterophylla (Schrad.) Dumort. & - & + & + & WR \\
\hline Marchantia polymorpha L. & + & - & + & St, StA, SO \\
\hline Pellia neesiana (Gottsche) Limpr. & + & - & - & so \\
\hline $\begin{array}{l}\text { Plagiochila porelloides (Torr. ex Nees) } \\
\text { Lindenb. }\end{array}$ & + & - & + & SO, WR \\
\hline \multicolumn{5}{|c|}{ Bryophyta } \\
\hline Abietinella abietina (Hedw.) Fleisch. & + & - & - & SO \\
\hline Acaulon muticum (Hedw.) H. Müll. & - & + & - & SO, SV \\
\hline Amblystegium juratzkanum Schimp. & - & - & + & WR, WL \\
\hline Amblystegium serpens (Hedw.) Schimp. & + & + & + & St, StA, SO, WR, WL \\
\hline Anomodon attenuatus (Hedw.) Huebener & - & - & + & WL \\
\hline $\begin{array}{l}\text { Atrichum angustatum (Brid.) Bruch et } \\
\text { Schimp. }\end{array}$ & - & - & + & so \\
\hline Atrichum undulatum (Hedw.) P. Beauv. & + & + & + & SO, SG \\
\hline Aulacomnium palustre (Hedw.) Schwaegr.* & - & - & + & so \\
\hline Barbula unguiculata Hedw. & - & + & + & St, StA, SO, SG \\
\hline $\begin{array}{l}\text { Brachytheciastrum velutinum (Hedw.) } \\
\text { Ignatov et Huttunen }\end{array}$ & + & + & + & St, StA, SO, WR, WL \\
\hline Brachythecium campestre (H. Müll.) Schimp. & + & - & + & WR, WL \\
\hline $\begin{array}{l}\text { Brachythecium glareosum (Bruch ex Spruce) } \\
\text { Schimp. }\end{array}$ & + & - & + & $\begin{array}{c}\text { St, StA, SO, SV, WR, } \\
\text { WL }\end{array}$ \\
\hline Brachythecium rutabulum (Hedw.) Schimp. & + & - & + & St, StA, SO, WR, WL \\
\hline $\begin{array}{l}\text { Brachythecium salebrosum (Hoffm. ex Web. } \\
\text { et Mohr) Schimp. }\end{array}$ & + & + & + & St, StA, WR, WL \\
\hline $\begin{array}{l}\text { Brachythecium tommasinii (Sendt. ex Boulay) } \\
\text { Ignatov et Huttunen }\end{array}$ & + & - & + & SO, WR, WL \\
\hline $\begin{array}{l}\text { Bryoerythrophyllum recurvirostrum (Hedw.) } \\
\text { Chen }\end{array}$ & - & + & + & StA, SO \\
\hline
\end{tabular}

ISSN 1996-4536 (print) • ISSN 2311-0783 (on-line) • Біологічні Студії / Studia Biologica • 2018 • Том 12/№1 • C. 99-112 
Continued Table 2

\begin{tabular}{|c|c|c|c|c|}
\hline 1 & 2 & 3 & 4 & 5 \\
\hline Bryum argenteum Hedw. & - & + & + & St, StA, SG \\
\hline Bryum caespiticium Hedw. & + & + & + & St, SO \\
\hline Bryum dichotomum Hedw. & + & - & - & St, StA \\
\hline Bryum subapiculatum Hampe & - & - & + & StA \\
\hline Callicladium haldanianum (Grev.) Crum & + & + & - & so \\
\hline Calliergon cordifolium (Hedw.) Kindb.* & - & - & + & So \\
\hline Calliergonella cuspidata (Hedw.) Loeske & + & - & + & $\mathrm{SO}, \mathrm{AM}$ \\
\hline Calliergonella lindbergii (Mitt.) Hedenäs & - & - & + & StA \\
\hline Campylium sommerfeltii (Myrin) Lange & - & + & + & St, WR \\
\hline Ceratodon purpureus (Hedw.) Brid. & + & + & + & St, StA, SO, SG, WL \\
\hline Cirriphyllum piliferum (Hedw.) Grout & + & - & - & so \\
\hline Climacium dendroides (Hedw.) Weber et Mohr & - & + & - & SO \\
\hline Cratoneuron filicinum (Hedw.) Spruce & - & - & + & St, StA, SO, AM \\
\hline Dicranella heteromalla (Hedw.) Schimp. & + & + & + & SO, SG, WL \\
\hline Dicranella schreberiana (Hedw.) Dix. & - & + & - & SO \\
\hline Dicranodontium denudatum (Brid.) Britton & - & + & - & so \\
\hline Dicranum montanum Hedw. & - & - & + & St, SG, SO, WL \\
\hline Dicranum scoparium Hedw. & + & + & - & SO \\
\hline Didymodon fallax (Hedw.) Zander & - & - & + & StA \\
\hline Didymodon rigidulus Hedw. & - & + & + & St, StA, SO \\
\hline Didymodon vinealis (Brid.) Zander & - & - & + & StA \\
\hline Diphyscium foliosum (Hedw.) Mohr & - & - & + & so \\
\hline Ditrichum heteromallum (Hedw.) Britton & + & - & - & so \\
\hline Drepanocladus aduncus (Hedw.) Warnst. & - & - & + & St, SO, WR \\
\hline Encalypta streptocarpa Hedw. & - & + & + & so \\
\hline Entosthodon fascicularis (Hedw.) H. Müll. & - & + & + & SO \\
\hline Ephemerum serratum (Hedw.) Hampe & - & + & - & SO, SV \\
\hline $\begin{array}{l}\text { Eucladium verticillatum (With.) Bruch et } \\
\text { Schimp. }\end{array}$ & - & - & + & StA \\
\hline Eurhynchium angustirete (Broth.) T. Kop. & + & - & + & SO, WL \\
\hline Eurhynchium striatum (Hedw.) Schimp. & + & - & + & SO, SV \\
\hline Fissidens bryoides Hedw. & - & + & + & so \\
\hline Fissidens exilis Hedw. & - & - & + & SO \\
\hline Fissidens taxifolius Hedw. & + & + & + & so \\
\hline Funaria hygrometrica Hedw. & - & + & + & StA, SO, SV \\
\hline Grimmia pulvinata (Hedw.) Sm. & - & + & + & St, StA \\
\hline
\end{tabular}

ISSN 1996-4536 (print) •ISSN 2311-0783 (on-line) • Біологічні Студії / Studia Biologica • 2018 • Том 12/№1 • C. 99-112 
Continued Table 2

\begin{tabular}{|c|c|c|c|c|}
\hline 1 & 2 & 3 & 4 & 5 \\
\hline Herzogiella seligeri (Brid.) Iwats. & + & - & - & so \\
\hline Homalia trichomanoides (Hedw.) Brid. & - & + & - & WL \\
\hline $\begin{array}{l}\text { Homomallium incurvatum (Schrad. ex Brid.) } \\
\text { Loeske }\end{array}$ & + & - & + & St, StA \\
\hline $\begin{array}{l}\text { Hygroamblystegium humile (P. Beauv.) } \\
\text { Vanderp., Goffinet \& Hedenäs }\end{array}$ & - & - & + & St, StA, SO \\
\hline Hygroamblystegium varium (Hedw.) Mönk. & - & - & + & StA, SO, SG, WR, WL \\
\hline Hylocomium splendens (Hedw.) Schimp. & + & + & - & StA, SO \\
\hline Hypnum cupressiforme Hedw. & + & - & + & StA, SO, WR, WL \\
\hline Hypnum imponens Hedw. & - & - & + & so \\
\hline Hypnum pallescens (Hedw.) P.Beauv. & + & - & + & St, SO, WL \\
\hline Hypnum recurvatum (Lindb. et Arnell) Kindb. & - & - & + & StA, SO \\
\hline Leptodictyum riparium (Hedw.) Warnst. & - & - & + & StA, WR, AM \\
\hline Leskea polycarpa Hedw. & - & + & + & WL \\
\hline Leucobryum glaucum (Hedw.) Ångstr. & + & + & - & SO, SG \\
\hline Leucodon sciuroides (Hedw.) Schwägr. & - & - & + & WL \\
\hline Mnium hornum Hedw. & - & + & + & SO, SV, WL \\
\hline Mnium lycopodioides Schwägr. & - & - & + & SO \\
\hline Mnium marginatum (Dicks.) P. Beauv. & + & - & - & so \\
\hline Mnium spinosum (Voit) Schwägr. & - & - & + & so \\
\hline Mnium thomsonii Schimp. & + & - & + & SO \\
\hline $\begin{array}{l}\text { Nyholmiella obtusifolia (Schrad. ex Brid.) } \\
\text { Holmen et E. Warncke }\end{array}$ & - & - & + & WL \\
\hline Orthotrichum affine Schrad. ex Brid. & - & - & + & WL \\
\hline Orthotrichum anomalum Hedw. & - & + & + & StA, WR \\
\hline Orthotrichum cupulatum Hoffm. ex Brid. & - & + & - & WL \\
\hline Orthotrichum diaphanum Schrad. ex Brid. & - & - & + & StA, WL \\
\hline Orthotrichum lyellii Hook. et Taylor & - & - & + & WL \\
\hline Orthotrichum pallens Bruch ex Brid. & - & - & + & WL \\
\hline Orthotrichum pumilum Sw. & - & - & + & WL \\
\hline Orthotrichum schimperi Hammar & - & - & + & WL \\
\hline Orthotrichum speciosum Nees & - & - & + & WL \\
\hline Oxyrrhynchium hians (Hedw.) Loeske & + & + & + & St, StA, SO, SV, WL \\
\hline Physcomitrium eurystomum Sendtn. & - & + & - & so \\
\hline $\begin{array}{l}\text { Physcomitrium pyriforme (Hedw.) Bruch } \\
\text { et Schimp. }\end{array}$ & - & - & + & SO, SG \\
\hline
\end{tabular}

ISSN 1996-4536 (print) •ISSN 2311-0783 (on-line) • Біологічні Студії / Studia Biologica • 2018 • Том 12/№1 • C. 99-112 
Continued Table 2

\begin{tabular}{|c|c|c|c|c|}
\hline 1 & 2 & 3 & 4 & 5 \\
\hline Plagiomnium cuspidatum (Hedw.) T. Kop. & + & - & + & SO, SV, WL \\
\hline Plagiomnium ellipticum (Brid.) T. Kop. & + & - & + & SO, WR \\
\hline $\begin{array}{l}\text { Plagiomnium medium (Bruch et Schimp.) } \\
\text { T. Kop.* }\end{array}$ & - & - & + & SO \\
\hline Plagiomnium rostratum (Schrad.) T. Kop. & - & - & + & St, SO \\
\hline Plagiomnium undulatum (Hedw.) T. Kop. & - & - & + & SO, SG \\
\hline Plagiothecium cavifolium (Brid.) Iwats. & + & - & + & SO, WL \\
\hline $\begin{array}{l}\text { Plagiothecium curvifolium Schlieph. } \\
\text { ex Limpr. }\end{array}$ & - & + & - & so \\
\hline Plagiothecium denticulatum (Hedw.) Schimp. & + & + & - & SO \\
\hline Plagiothecium laetum Schimp. & + & - & - & so \\
\hline Plagiothecium latebricola Schimp. & - & - & + & So \\
\hline Plagiothecium nemorale (Mitt.) Jaeg. & + & + & + & SO, WL \\
\hline Plagiothecium succulentum (Wils.) Lindb. & - & - & + & St, SO \\
\hline Platygyrium repens (Brid.) Schimp. & - & - & + & St, WR, WL \\
\hline Pleurozium schreberi (Willd. ex Brid.) Mitt. & + & - & - & So \\
\hline Pogonatum urnigerum (Hedw.) P. Beauv. & - & + & - & SO \\
\hline Pohlia nutans (Hedw.) Lindb. & + & - & + & SO, WL \\
\hline Polytrichum formosum Hedw. & + & + & + & so \\
\hline Polytrichum juniperinum Hedw. & - & + & - & so \\
\hline Polytrichum longisetum Sw. ex Brid. & + & + & - & so \\
\hline Polytrichum piliferum Hedw. & - & + & - & SO \\
\hline Pseudoleskeella nervosa (Brid.) Nyh. & - & - & + & St, WL \\
\hline Pseudoscleropodium purum (Hedw.) Fleisch. & + & - & - & SO \\
\hline $\begin{array}{l}\text { Ptychostomum capillare (Hedw.) Holyoak } \\
\text { et N. Pedersen }\end{array}$ & - & - & + & SO, WR \\
\hline $\begin{array}{l}\text { Ptychostomum creberrimum (Taylor) } \\
\text { J. R. Spence et H. P. Ramsay }\end{array}$ & - & - & + & StA \\
\hline $\begin{array}{l}\text { Ptychostomum moravicum (Podp.) Ros } \\
\text { et Mazimpaka }\end{array}$ & - & - & + & StA, WR, WL \\
\hline $\begin{array}{l}\text { Ptychostomum pseudotriquetrum (Hedw.) } \\
\text { J. R. Spence et H. P. Ramsay }\end{array}$ & - & - & + & StA \\
\hline $\begin{array}{l}\text { Ptychostomum rubens (Mitt.) Holyoak } \\
\text { et N. Pedersen }\end{array}$ & - & - & + & SV \\
\hline Pylaisia polyantha (Hedw.) Schimp. & - & + & + & St, StA, SO, WR, WL \\
\hline Rhizomnium punctatum (Hedw.) T. Kop. & + & + & + & St, StA, SO, WR, WL \\
\hline Rhynchostegium confertum (Dicks.) Schimp. & - & - & + & SO \\
\hline Rhynchostegium murale (Hedw.) Schimp. & - & + & + & St, StA, SO \\
\hline
\end{tabular}

ISSN 1996-4536 (print) • ISSN 2311-0783 (on-line) • Біологічні Студії / Studia Biologica • 2018 • Том 12/№1 • С. 99-112 
End of the Table 2

\begin{tabular}{|c|c|c|c|c|}
\hline 1 & 2 & 3 & 4 & 5 \\
\hline $\begin{array}{l}\text { Rhytidiadelphus squarrosus (Hedw.) } \\
\text { Warnst. }\end{array}$ & - & + & + & SO \\
\hline Rhytidiadelphus triquetrus (Hedw.) Warnst. & - & + & - & so \\
\hline $\begin{array}{l}\text { Schistidium apocarpum (Hedw.) Bruch } \\
\text { et Schimp. }\end{array}$ & - & - & + & St, StA, WL \\
\hline Schistidium crassipilum Blom & - & + & + & St, StA \\
\hline $\begin{array}{l}\text { Sciuro-hypnum populeum (Hedw.) Ignatov } \\
\text { et Huttunen }\end{array}$ & - & - & + & St, StA, SG, WR \\
\hline $\begin{array}{l}\text { Sciuro-hypnum reflexum (Starke) Ignatov } \\
\text { et Huttunen }\end{array}$ & - & - & + & St, StA, SO, WL \\
\hline $\begin{array}{l}\text { Sciuro-hypnum starkei (Brid.) Ignatov } \\
\text { et Huttunen }\end{array}$ & - & - & + & StA \\
\hline $\begin{array}{l}\text { Straminergon stramineum (Dicks. ex Brid.) } \\
\text { Hedenäs* }\end{array}$ & - & - & + & SO \\
\hline Syntrichia papillosa (Wils.) Jur. & - & - & + & WL \\
\hline Syntrichia ruralis (Hedw.) F. Weber et Mohr & - & + & + & StA, SO \\
\hline Syntrichia virescens (De Not.) Ochyra & - & + & + & StA, SO, WL \\
\hline Tetraphis pellucida Hedw. & - & + & + & SO, WR \\
\hline Thuidium assimile (Mitt.) Jaeg. & + & - & - & so \\
\hline Thuidium delicatulum (Hedw.) Schimp. & + & - & - & SO \\
\hline Thuidium recognitum (Hedw.) Lindb. & + & - & - & SO \\
\hline Tortula acaulon (With.) R.H. Zander & - & + & + & SO, SG \\
\hline Tortula caucasica Lindb. ex Broth. & - & + & - & so \\
\hline Tortula muralis Hedw. & - & + & + & St, StA \\
\hline Tortula protobryoides R.H. Zander & - & + & - & so \\
\hline Tortula subulata Hedw. & - & + & + & SO, SV \\
\hline Trichodon cylindricus (Hedw.) Schimp. & - & - & + & StA \\
\hline
\end{tabular}

Comments: 1900-1940 - materials of the Herbarium of Ivan Franko National University of Lviv (LW) (FI. Lilienfeld, H. Drozdovskaya); 1941-1990 - data of Herbarium of National Museum of Natural History of the NAS of Ukraine (LWS) and of Institute of the Ecology of the Carpathians of the NAS of Ukraine (LWKS) (collection and definition of briologists: M. Slobodian, A. Lazarenko, K. Ulychna, I. Danylkiv); 1991-2017 - collection and definition of bryophyte authors of the article, * - literary data $[1 ; 2 ; 25 ; 31 ; 32]$

St - stony; StA - stony artificial; SG - soil with gravel or sand; SO - soil; SV - on the soil among the grass plants or leaf litter; WR- rotten wood; WL - living wood; AM - aqueous medium

Примітки: 1900-1940 - матеріали Гербарію ЛНУ ім. Івана Франка (Фл. Лілієнфрельд, Г. Дроздовської); 1941-1990 - дані Гербаріїв ДПМ НАНУ й Інституту екології Карпат НАНУ (збори і визначення бріологів: М. Слободяна, А. Лазаренка, К. Уличної, І. Данилківа); 1991-2017 - збори і визначення мохоподібних авторами статті, * - літературні дані $[1 ; 2 ; 25 ; 31 ; 32]$

St - кам'янисті, StA - штучні кам'янисті, SG - ґрунт із гравієм або піщаний, SO - оголений ґрунт, SV - серед трав'яних рослин на ґрунті; WR - гнила деревина, WL - форофіти, AM водне середовище

ISSN 1996-4536 (print) • ISSN 2311-0783 (on-line) • Біологічні Студії / Studia Biologica • 2018 • Том 12/№1 • C. 99-112 
Along with the disappearance of some species of bryophyte, one can observe the reduction of frequency of occurrence in other species. For example, the species Eurhynchium angustirete, E. striatum, Brachythecium tommasinii which are often found in herbarium specimens, occur today.

The species Leucodon sciuroides is considered to be very sensitive to atmospheric air pollution $\left(\mathrm{SO}_{2}\right)$, and its disappearance was recorded in many cities [7; 18; 37]. However, it has recently restored its population in some areas [21;36], or grows, like other epiphytes Anomodon attenuatus, Orthotrichum lyellii in relatively little disturbed ecotope [24].

Anthropogenic transformation of bryoflora also manifested itself in the fact that a number of bryophyte species disappeared as a result of indigenous vegetation changes. The loss of the corresponding ecotopes, first of all in rotten wood, has caused a relatively small variety of liverworts which is characteristic for many cities and urban parks [3; 23; 24; 29; 35]. Some species appeared due to on increase of some technogenic substrates, as well as the possible loss of competitors i.e. other bryophytes from the same ecological niches [16]. An increasing anthropogenic ecotopes number, in particular, stony which eventually is populated by bryophytes is a consequence of urban processes. For bryophytes this is manifested in a significant increasing of the part of the epilithic species [10; 19; 33].

According to the substrate preference, the highest number are the epigeic: 103 species (72\%) which most often settle in the naked soil (SO) - 100 species, a very small percentage occur on sandy soil or gravel (SG) - 12 species $(8.4 \%)$, among herbaceous plants on the soil (SV) - 11 species $(7.7 \%$ ) (Table 2). Three species grow in water (AM) (2\%), epixylic species (WR) $-21(15 \%)$, and 4 species among the last ones were found only on rotten wood.

55 species $(38.5 \%)$ were found on stony substrates, among them 35 species of epilithes were registered on the corresponding natural substrates and 45 species - on the substrates of artificial origin. In general, 9 species were found only on artificial stone substrates $(6.3 \%)$.

43 species of epiphytes $(30.1 \%)$ were found on the territory of the study. In recent decades, the growth of colonisation indices of phorophytes by the epiphytic mosses, primarily by the urbanophilic mosses and urbanotolerant species have been recorded in the cities: Leskea polycarpa, Pylaisia polyantha, Platygyrium repens, Amblystegium serpens, Hygroamblystegium varium, Brachythecium salebrosum, Orthotrichum pumilum, O. diaphanum, Ptychostomum moravicum. They are typical for urboecosystem of Lviv [17; 18], often occur on "Pohulyanka" area and some of them, besides bark of living trees, populate other substrates. This nucleus of urbanophilic species "briofitos urbanos" [27; 28] is worth to be supplemented with species mainly cosmopolitan: Atrichum undulatum, Barbula unguiculata, Brachythecium rutabulum, Bryum argenteum, B. caespiticium, Ceratodon purpureus, Didymodon rigidulus, Funaria hygrometrica, Marchantia polymorpha, Oxyrrhynchium hians, Plagiomnium cuspidatum, Schistidium apocarpum, Syntrichia ruralis, Tortula subulata which are also common for many cities [3; 7-9; 11$13 ; 23 ; 24 ; 26 ; 29 ; 33 ; 37]$.

In contrast to the central part of the city of Lviv where 30 species of bryophytes [19] were found or to the ecotopes of the Lviv railways (41 species) [20], "Pohulyanka" is characterized by significant bryophlora diversity, because natural and semi-natural ecotopes were preserved: the waterlogged places near the springs and the reservoirs, the slopes of the ravines, which hawe undergone much less recreational press and damage.

ISSN 1996-4536 (print) • ISSN 2311-0783 (on-line) • Біологічні Студії / Studia Biologica • 2018 • Том 12/№1 • С. 99-112 
At the same time, over the past decades, it has been registered the decline of forest species, including rare species of Forest and Forest-Steppe zones which have disappeared in the forest park area (Acaulon muticum, Pogonatum urnigerum, Leucobryum glaucum, Rhytidiadelphus triquetrus).

1. Bachuryna H.F., Melnychuk V.M. Moss Flora of the Ukrainian SSR. Kyiv: Naukova Dumka, 1987. V. 1. 180 p.; 1988. V. 2. 180 p.; 1989. V. 3. 176 p. (In Ukrainian).

2. Bachuryna H.F., Melnychuk V.M. Moss Flora of the Ukraine. Kyiv: Naukova Dumka, 2003; 4. 255 p. (In Ukrainian).

3. Barsukov O.O. Bryoflora of urban territories of Kharkiv oblast. Chornomors'k. bot. z, 2014; 10(3): 305-321. doi:10.14255/2308-9628/14.103/3. (In Ukrainian).

4. Boiko M.F. Red List of Bryobionta of Ukraine. Rare and endangered species of the Bryobionta of Ukraine. Kherson: Ailant, 2010. 94 p. (In Ukrainian).

5. Boiko M.F. The Second checklist of Bryobionta of Ukraine. Chornomors'k. bot. z, 2014; 10(4): 426-487. doi:10.14255/2308-9628/14.104/2.

6. Boiko M.F. Materials to the Red Data Book of Ukraine (Sphagnopsida, Bryopsida). Chornomors'k. bot. z, 2015; 11 (4): 449-502. doi:10.14255/2308-9628/15.114/5. (In Ukrainian).

7. Dymytrova L. Epiphytic lichens and bryophytes as indicators of air pollution in Kyiv city (Ukraine). Folia Cryptog. Estonica, Fasc, 2009; 46: 33-44.

8. Filipiak E., Sieradzki J. Wstepne badania nad brioflora Lodzi. Fragmenta Floristica et Geobotanica. Series: Polonica, 1996; 3: 117-129.

9. Fojcik B., Stebel A. The diversity of moss flora of Katowice town (S. Poland). Cryptogamie, Bryologie, 2014; 35(4): 373-385.

10. Fudali $E$. Influence of city on the floristical and ecological diversity of bryophytes in parks and cemeteries. Biodivers. Res. Conservation, 2006; 1-2: 131-137.

11. Fudali E. Contemporary changes in urban bryophytes; a case study of Wrociaw parks carried out in 2000, 2006 and 2011). Acta Botanica Silesiaca, 2012; 6: 81-95.

12. Gapon Yu V. The list of bryophytes of Poltava city and its surroundings. Biology and Ecology, 2016; 2(2): 40-50.

13. Godovičová K., Mišíková K. Epifytické machorasty urbánneho prostredia Bratislavy. Epiphytic bryophytes in the urban environment of Bratislava. Bryonora, 2017; 59: 44-57.

14. Hodgetts N. G. Checklist and country status of European bryophytes - towards a new Red List for Europe. Irish Wildlife Manuals, 2015; 84. 125 p.

15. Kucheravyj V. P. Gardens and parks of Lviv city. Lviv: Publishing House "Svit", 2008. 124132. (In Ukrainian).

16. Mamchur Z. Antropogenic transformation of the epiphytic bryoflora of Lviv city and surroundings. Visnyk of Lviv Univ. Biology series, 2003; 34: 135-141. (In Ukrainian).

17. Mamchur Z. Urbanophilic epiphytic mosses of Lviv city. Visnyk of the Lviv University. Series Biology, 2010; 54: 115-122. (In Ukrainian).

18. Mamchur Z., Bilska I. Epiphytic bryophytes features in urban environment. Visnyk of the Lviv University. Series Biology, 2013; 61: 125-132. (In Ukrainian).

19. Mamchur Z., Chuba M. The ecological features of synanthropic flora of dense housing area of Lviv. Studia Biologica, 2016. 10(1): 143-154. (In Ukrainian).

20. Mamchur Z., Chuba M., Drach Yu. Mosses and vascular plants on railway tracks in the Lviv city. Visnyk of the Lviv University. Series Biology, 2017; 75: 54-65. (In Ukrainian).

21. Pescott O., Preston C. Rare and interesting. Field Bryology, 2014; 111: 42-45.

22. Red Data Book of European Bryophytes. Trondheim, The European Committee for Conservation of Bryophytes, 1995. 291 p.

23. Sabovljević M., Grdović S. Bryophyte Diversity Within Urban Areas: Case Study of the City of Belgrade (Serbia). International Journal of Botany, 2009; 5: 85-92.

ISSN 1996-4536 (print) • ISSN 2311-0783 (on-line) • Біологічні Студії / Studia Biologica • 2018 • Том 12/№1 • C. 99-112 
24. Sabovljević M., Sabovljević A. Biodiversity within urban areas: A case study on bryophytes of the city of Cologne (NRW, Germany). Plant Biosystems, 2009; 143: 3: 473-481.

25. Scherbachenko O., Rabyk I. Bryophytes of the side zone of Lviv reservoirs. Proc. of the State Nat. Hist. Museum, Lviv, 2004; 19: 39-46. (In Ukrainian).

26. Skudnik M., Sabovljević A., Batič F., Sabovljević M. The bryophyte diversity of Ljubljana (Slovenia). Polish Botanical Journal, 2013; 58(1): 319-324. [DOI: 10.2478/pbj-2013-0031]

27. Soria A., Ron M. Datos para el conocimiento de la flora briolygica urbana de la ciudad de Logroco. Anales Jard. Bot. Madrid, 1990; 46(2): 427-432.

28. Soria A., Ron M. Aportaciones al conocimiento de la brioflora urbana espacola. Cryptogamie, 1995; 16(4): 285-299.

29. Szücs $P$. Bryophyte flora of the Botanic Garden of the University of Sopron (W Hungary). Studia Bot. Hung, 2017; 48(1): 77-88. [DOI: 10.17110/StudBot.2017.48.1.77]

30. Tasenkevich L., Mamchur Z., Khmil T., Zhuk O. Personal collections (XIX-XX centuries) in the Herbarium of Ivan Franko National University of Lviv (LW). Visnyk of the Lviv University. Series Blology, 2014; 65: 112-120. (In Ukrainian).

31. Ulychna K.O. The leafy mosses I. II. In the book: Catalog of museum funds. State Natural History Museum of the Academy of Sciences of the Ukrainian SSR. Kyiv: Naukova Dumka, 1978. P. 57-73. (In Ukrainian).

32. Ulychna K.O. Herbarium of mosses. The leafy mosses III. In the book: Catalog of museum funds. State Natural History Museum of the Academy of Sciences of the Ukrainian SSR. Kyiv: Naukova Dumka, 1979. 4-18. (In Ukrainian).

33. Virchenko V.M. Bryophytes of the forest-park zone of Kyiv. Kyiv: Znannya Ukrainy, 2006. 32 p. (In Ukrainian).

34. Virchenko V.M., Pryadko O.I., Onyshchenko V.A. Bryophytes / Vascular plants and bryophytes of Holosiivskyi national nature park. Kyiv: Alterpress, 2016. P. 66-85. (In Ukrainian).

35. Wolski G.J., Stefaniak A., Kowalkiewicz B. Bryophytes of the experimental and teaching garden of the faculty of biology and environmental protection, university of Lodz (Poland). Ukr. Bot. J, 2012; 69(4): 519-529.

36. Zechmeister H.G., Tribsch A., Hohenwallner D. Die Moosflora von Linz und ihre Bedeutung für die Bioindikation. Naturkundliches Jahrbuch der Stadt Linz, 2002; 48: 111-191.

37. Zechmeister H.G., Grodzicska K., Szarek-Jukaszewska G. Bryophytes / Bioindicators and biomonitors / B.A. Markert, A.M. Breure, H.G. Zechmeister. Elsevier Science Ltd., 2003; 6(1 $1^{\text {st }}$ Ed.): $329-375$.

\title{
МОХОПОДІБНІ ЛІСОПАРКУ “ПОГУЛЯНКА” (м. ЛЬВІВ). \\ І. ЗМІНИ У ТАКСОНОМІЧНОМУ СКЛАДІ ЗА ВПЛИВУ АНТРОПОГЕННОЇ ТРАНСФОРМАЦІї
}

\author{
3. Мамчур, Ю. Драч, І. Данилків \\ Львівський національний університет імені Івана Франка \\ вул. Грушевського, 4, Львів 79000, Україна \\ e-mail: dzvinkamamchur@gmail.com
}

На основі матеріалів власних польових досліджень, аналізу матеріалів Гербаріїв (LW, LWS, LWKS), а також літературних даних для території лісопарку “Погулянка", у м. Львові складено список видів мохоподібних, який охоплює 143 види, що належать до 79 родів, 36 родин із двох відділів: Marchantiophyta i Bryophyta. Проаналізовано зміни у видовому складі за останні 50-100 років: за період досліджень (1991-2017) не виявлено 34 видів, відзначено зменшення видового 
різноманіття печіночників, зменшення частки родин: Polytrichaceae Schwägr., Thuidiaceae Schimp., Hylocomiaceae M. Fleisch. Найменше змін зазнали родини Brachytheciaceae Schimp., Hypnaceae Schimp., Mniaceae Schwägr., а в родинах Amblystegiaceae Kindb., Bryaceae Schwägr., Orthotrichaceae Arn., Mniaceae Schwägr. спостерігаємо збільшення числа видів. Уперше для досліджуваної території вказано 72 види бріофрітів, Eucladium verticillatum (With.) Bruch et Schimp. - для території Львівської області. 25 видів $є$ раритетними для Неморальної та Лісостепової зон, із них за останні десятиліття на території Погулянки вперше виявлено регіонально рідкісні види: Bryum subapiculatum Hampe, Diphyscium foliosum (Hedw.) Mohr, Eucladium verticillatum, Fissidens exilis Hedw., Orthotrichum lyelli Hook. et Tayl., Rhynchostegium confertum (Dicks.) Schimp., Sciuro-hypnum starkei (Brid.) Ignatov et Huttunen, Trichodon cylindricus (Hedw.) Schimp.

Подано субстратну приналежність бріофітів: найбільшу кількість становлять епігейні види (72 \%), значний відсоток епілітних (38,5 \%) і епіфітних видів $(30,1 \%)$, натомість незначна кількість епіксилів (16,7 \%). 3'ясовано, що для території лісопарку “Погулянка” властиве значне бріофлористичне розмаїття, на відміну від центральної частини міста Львова чи території залізниці, оскільки збереглися природні й напівприродні екотопи. 3'ясовано, що внаслідок антропогенної діяльності відбулося випадання лісових видів, натомість завдяки збільшенню кількості антропогенних субстратів (зокрема, кам'янистих) зросла частка епілітних видів.

Ключові слова: мохоподібні, антропогенна трансформація, регіонально рідкісні види, лісопарк “Погулянка”, м. Львів

Одержано: 10.01.2018

ISSN 1996-4536 (print) • ISSN 2311-0783 (on-line) • Біологічні Студії / Studia Biologica • 2018 • Том 12/№1 • C. 99-112 